\title{
Article \\ Molecular Recognition by Silicon Nanowire Field-Effect Transistor and Single-Molecule Force Spectroscopy
}

\author{
Francisco M. Espinosa, Manuel R. Uhlig (D) and Ricardo Garcia *(D) \\ Instituto de Ciencia de Materiales de Madrid, Consejo Superior de Investigaciones Cientificas, \\ c/ Sor Juana Inés de la Cruz 3, 28049 Madrid, Spain; francisco.espinosa@csic.es (F.M.E.); \\ manuel.uhlig@csic.es (M.R.U.) \\ * Correspondence: r.garcia@csic.es
}

check for updates

Citation: Espinosa, F.M.; Uhlig, M.R.; Garcia, R. Molecular Recognition by Silicon Nanowire Field-Effect Transistor and Single-Molecule Force Spectroscopy. Micromachines 2022, 13, 97. https://doi.org/10.3390/ mi13010097

Academic Editors: Amalio Fernández-Pacheco and Javier Pablo-Navarro

Received: 14 December 2021

Accepted: 5 January 2022

Published: 8 January 2022

Publisher's Note: MDPI stays neutral with regard to jurisdictional claims in published maps and institutional affiliations.

Copyright: (c) 2022 by the authors. Licensee MDPI, Basel, Switzerland. This article is an open access article distributed under the terms and conditions of the Creative Commons Attribution (CC BY) license (https:// creativecommons.org/licenses/by/ $4.0 /)$.

\begin{abstract}
Silicon nanowire (SiNW) field-effect transistors (FETs) have been developed as very sensitive and label-free biomolecular sensors. The detection principle operating in a SiNW biosensor is indirect. The biomolecules are detected by measuring the changes in the current through the transistor. Those changes are produced by the electrical field created by the biomolecule. Here, we have combined nanolithography, chemical functionalization, electrical measurements and molecular recognition methods to correlate the current measured by the SiNW transistor with the presence of specific molecular recognition events on the surface of the SiNW. Oxidation scanning probe lithography (o-SPL) was applied to fabricate sub-12 nm SiNW field-effect transistors. The devices were applied to detect very small concentrations of proteins (500 pM). Atomic force microscopy (AFM) single-molecule force spectroscopy (SMFS) experiments allowed the identification of the protein adsorption sites on the surface of the nanowire. We detected specific interactions between the biotinfunctionalized AFM tip and individual avidin molecules adsorbed to the SiNW. The measurements confirmed that electrical current changes measured by the device were associated with the deposition of avidin molecules.
\end{abstract}

Keywords: oxidation scanning probe lithography; silicon nanowire; field-effect transistor; singlemolecule force spectroscopy; AFM

\section{Introduction}

Different types of field-effect transistor were proposed to detect biomolecular processes [1-5]. Among them, silicon nanowire FETs offered a genuine nanoscale platform to detect biological molecules. Those devices have targeted different biomedical and biological applications as the specific detection of viruses, proteins, nucleic acids, cancer biomarkers, and cells or monitoring the physiological responses of a specific therapeutic treatment on cells or tissues [6-8].

Top-down approaches such as scanning probe lithography were applied to fabricated SiNW devices [9-11]. In particular, oxidation scanning probe lithography (o-SPL) was applied to fabricate biosensors [12]. Oxidation SPL combines the high-spatial resolution capabilities of atomic force microscopy (AFM) for positioning with the capability to fabricate nanoscale features $[13,14]$. It has been extensively applied in nanopatterning and for the fabrication of a variety of nanoelectronics devices [10-29].

Here, oxidation SPL, silicon nanowire transistors and single-molecule force spectroscopy are combined to quantify the molecular recognition events happening on the surface of a silicon nanowire biosensor. The SiNW field-effect transistor detected very low concentrations of proteins (500 pM). We demonstrated the existence of specific biomolecule interactions on the surface of the SiNW. Those interactions were associated with the presence of avidin molecules. The study aimed to bridge the gap between the indirect measurements of biomolecular activity provided by nanoscale transistors and the direct measurements provided by single-molecule force spectroscopy. 


\section{Materials and Methods}

\subsection{Silicon Nanowires}

The silicon nanowires were made by o-SPL on substrates from ultra-thin silicon on insulator (SOI) wafer (MEMC/SunEdison, Belmont, CA, USA). The top (100)-oriented Si layer is $12 \mathrm{~nm}$ thick, p-doped, has a nominal resistivity of 9-15 $\Omega \mathrm{cm}$ and the buried oxide layer (BOX) has $25 \mathrm{~nm}$ of thickness. The substrates were cleaned with a protocol that involves three sonication cycles of 10 min each in a mixture of $\mathrm{NH}_{4} \mathrm{OH}-\mathrm{H}_{2} \mathrm{O}_{2}-\mathrm{H}_{2} \mathrm{O}(1: 1: 5)$. Then, the samples are sonicated for $5 \mathrm{~min}$ in deionized water.

Oxidation SPL was applied to generate ultrathin and narrow silicon oxide masks. It was performed at a relative humidity of $45 \%$. We have used $n+$-type doped silicon cantilevers (NCHV-W, Bruker) with a force constant of about $42 \mathrm{~N} \mathrm{~m}^{-1}$ and a resonant frequency of $320 \mathrm{kHz}$. The cantilever was excited at its resonant frequency. Typical voltage pulses were of 21-27 V with 0.7-1 ms duration. General and detailed aspects about o-SPL might be found elsewhere [14]. The SiNWs used here were 10-12 nm in thickness, $150 \mathrm{~nm}$ in width and $5 \mu \mathrm{m}$ in length.

In a later stage, the ultrathin oxide mask was removed by applying reactive ion etching (NRE 300 RIE System de NANO-MASTER, Inc, Austin, TX, USA). The sample was introduced in the RIE chamber and the pressure lowered to $10^{-5}$ Torr for $30 \mathrm{~min}$. Then the $\mathrm{SF}_{6}-\mathrm{O}_{2}$ gas mixture $(10: 5 \mathrm{sccm})$ was introduced and left to stabilize for $1 \mathrm{~min}$ at the specific chamber pressure of the experiment $\left(6.25 \times 10^{-2}\right.$ Torr $)$. A $20 \mathrm{~W}$ radio frequency power was applied for $45 \mathrm{~s}$.

\subsection{Microelectrodes}

First, a layer of photoresist S1813 was deposited on the sample and centrifuged at $5000 \mathrm{rpm}$ for $1 \mathrm{~min}$. It was cured on a hot plate $\left(115^{\circ} \mathrm{C}\right)$ for $1 \mathrm{~min}$. The microelectrodes were patterned by UV photolithography. A UV mask was used to protect the transistor channel from light exposure (8s). Then, the sample was immersed in the developer (MF319) for 1 min, rinsed with deionized water and dried with $\mathrm{N}_{2}$. After that, the sample was treated with oxygen plasma for $30 \mathrm{~s}$ to eliminate the resist residues. To make the microelectrodes, a thin film $\mathrm{Cr}(5 \mathrm{~nm}) / \mathrm{Au}(40 \mathrm{~nm})$ was deposited by electron beam evaporation.

The electrodes were protected from the buffer by a thin film of polydimethylsiloxane (PDMS). A solution of PDMS and hexane (1:100) was centrifuged at $3000 \mathrm{rpm}$ for $30 \mathrm{~s}$. The resulting thickness was about $30 \mathrm{~nm}$. The sample was cured on the hotplate for $30 \mathrm{~min}$ at $90{ }^{\circ} \mathrm{C}$. In a later stage, the resist covering the channel was removed with acetone and rinsed with isopropanol and water. $\mathrm{An}_{2}$ plasma was used to remove the possible remains of organic residues from the surface of the microfluidic channel. To generate the $\mathrm{O}_{2}$, a power of $50 \mathrm{~W}$ was applied for $1 \mathrm{~min}$ in a chamber with a pressure of $0.5 \mathrm{mbar}$ (0.37 Torr).

\subsection{Chemicals}

Phosphate-buffered saline (PBS) powder, ethanol, 30\% hydrogen peroxide, $1 \mathrm{~N}(0.5 \mathrm{~mol} / \mathrm{L})$ sulfuric acid, 3-aminopropyltriethoxysilane (APTES) 99\%, trimethylamine $\geq 99 \%$ and avidin from egg white $\geq 98 \%$ were purchased from Sigma-Aldrich (Madrid, Spain). The N-Hydroxysuccinimid (NHS)-polyethylenglycol (PEG27) biotin linkers were purchased from JKU Linz [30].

\subsection{Samples Silanization with OTS}

Before octadecyltrichlorosilane (OTS) functionalization, the samples were exposed to an oxygen plasma. The conditions of the process were: $50 \mathrm{~W}, 0.4 \mathrm{mbar}$ and $30 \mathrm{~s}$. The samples were immediately immersed in $99.5 \%$ anhydrous toluene. In a glove box with an $\mathrm{N}_{2}$ atmosphere, the samples were passed from anhydrous toluene to a $5 \mathrm{~mL}$ solution of anhydrous toluene at $99.9 \%$ with $3 \mu \mathrm{L}$ of OTS for $105 \mathrm{~s}$. Then, the sample was rinsed with chloroform, ethanol and water, and dried with nitrogen. The sample with the OTS was cured on a hot plate at $80{ }^{\circ} \mathrm{C}$. 


\subsection{Proteins}

A freeze-dried avidin was dissolved in $1 \mathrm{mM} \mathrm{NaCl}$ to obtain a solution with an avidin concentration of $500 \mathrm{pM}$. Then, $100 \mu \mathrm{L}$ of the solution was deposited on the SiNW sample for $1 \mathrm{~min}$. Afterwards, the sample was rinsed carefully with $1 \mathrm{mM} \mathrm{NaCl}$ and subsequently with $20 \mathrm{mM}$ PBS.

\subsection{Tip Functionalization with APTES}

Silicon nitride cantilevers (MSCT, Bruker, Camarillo, CA, USA) were cleaned thoroughly using the RCA procedure. This procedure consisted of three subsequent baths in a mixture of ammonia solution, hydrogen peroxide (30\%) and ultrapure water (1:1:5 ratio in volume). Then, the cantilevers were rinsed with ultrapure water and dried carefully using a flow of $\mathrm{N}_{2}$. Afterwards, the cantilevers were exposed to oxygen plasma (Diener Electronic, Ebhausen, Germany) for $1 \mathrm{~min}$ at a power of $100 \mathrm{~W}$ under a pressure of $0.4 \mathrm{bar}$. Next, the cantilevers were transferred into a mixture of 3-aminopropyltriethoxysilane (APTES) and ethanol (1:5000 ratio in volume) to functionalize the tips. After $45 \mathrm{~min}$, the cantilevers were rinsed with ethanol and ultrapure water and dried with $\mathrm{N}_{2}$. Finally, the cantilevers were placed in a desiccator for $1 \mathrm{~h}$.

\subsection{Tip Functionalization with PEG-Biotin}

The functionalization of the tips with NHS-PEG27-Biotin linkers followed a similar protocol as described above. First, $1 \mathrm{mg}$ of the NHS-PEG27-Biotin linkers was dissolved in trichloromethane $(0.5 \mathrm{~mL})$. The obtained solution was transferred into a Teflon PTFE chamber and $30 \mu \mathrm{L}$ of tryethylamine were added as a catalyst. Then, the silanized tips were immersed into the chamber. After an incubation time of $2 \mathrm{~h}$, the cantilevers were removed from the chamber, rinsed three times with trichloromethane and dried with $\mathrm{N}_{2}$.

\subsection{Cantilever Calibration}

Cantilevers of the types PPP-NCH-W (Si, NanoWorld) and MSCT-C (SiN, Bruker) were used for AFM imaging. For the SMFS experiments, we used MSCT-D cantilevers $\left(\mathrm{SiN}_{\mathrm{x}}\right.$, Bruker). MSCT-D cantilevers were calibrated in liquid as follows: First, the inverse optical lever sensitivity (invOLS) for the static detection, $\sigma$, was obtained from force-displacement curves recorded on the gold electrodes. The invOLS $\sigma$ was determined as the inverse of each curve's slope in the contact part and then averaged over 256 curves. Second, the cantilever's thermal noise spectrum (power spectral density, PSD) was recorded at about $15 \mu \mathrm{m}$ above the sample surface [31]. Then, the single harmonic oscillator (SHO) model was fitted to the PSD around the peak of the first resonance frequency using the calculated invOLS of the first mode, $\sigma_{1}$ [32]. The fitting yields the force constant $k_{1}$. The static force constant can then be calculated by $k_{0}=k_{1} / 1.03$. The obtained values for the cantilevers used here were $k_{0}=32 \mathrm{pN} / \mathrm{nm}$ (Experiment 1) and $k_{0}=39 \mathrm{pN} / \mathrm{nm}$ (Experiment 2). The calibration was performed after the experiment to prevent tip damage during the calibration.

\subsection{AFM Experiments}

All the experiments were performed on a NanoWizard III AFM (JPK Instruments AG, Berlin, Germany) equipped with an open liquid cell. The experiments were carried out in $10 \mathrm{mM}$ PBS at pH 7.4 at a temperature of $T=302 \mathrm{~K}$. First, the SiNW was localized by AFM using an MSCT-C cantilever. Then, the position of the SiNW was marked in the optical image from the camera, and the laser spot was moved to the MSCT-D cantilever on the same chip. The new cantilever was then moved to the previously marked position and an SMFS measurement was started without further imaging. This procedure was developed to prevent damage of the tip functionalization due to AFM imaging. SMFS experiments were performed in the force-volume mode [33]. This method enabled us to directly correlate the obtained curves with the topography of the SiNW. The individual curves were acquired by applying a periodic trapezoidal modulation to the cantilever base with an amplitude of $A_{\mathrm{p}}=150 \mathrm{~nm}$. First, the cantilever base was brought towards the sample at a constant 
speed of $200 \mathrm{~nm} / \mathrm{s}$ until a force of $140 \mathrm{pN}$ was reached. This force value was chosen to avoid damage of the biotin. At this extension, the piezo was held for $200 \mathrm{~ms}$. Then, the cantilever base was retracted at a constant pulling speed of $v_{p}=1000 \mathrm{~nm} / \mathrm{s}$. FV images were performed on different SiNWs and covered different areas. For experiment 1 (2), it consisted of $20 \times 40(40 \times 60)$ force curves in an area of $0.5 \times 1.0 \mu^{2}\left(1.0 \times 1.5 \mu \mathrm{m}^{2}\right)$, resulting in a total of 800 (2400) force curves.

\subsection{Single-Molecule Force Spectroscopy (SMFS) Analysis}

All force-displacement curves were transformed into force-distance curves by $d=z+$ $F / k$. To detect the specific unbinding events, a custom written code was used (MATLAB, MathWorks, Natick, MA, USA) [34]. It fitted the retraction part of the SMFS curves with a polynomial function of grade 7 . Then, the minima of the polynomial were determined and the algorithm searched in their vicinity for minima in the raw data. Minima with a force value smaller than 1.5-fold of the baseline noise were disregarded to avoid spurious peaks. Specific unbinding events were selected by applying general criteria for specificity $[35,36]$. Only events that showed rupture distances $d_{\text {rup }}$ in the 5 to $17 \mathrm{~nm}$ range were considered. The above distance range matched the length of the PEG27 linker (10 nm $\pm 5 \mathrm{~nm}$ for the slightly shorter PEG24 [35]). In total, $<2 \%$ of the retraction curves exhibited features of specific unbinding events. Curves showing specific unbinding events were further processed using the JPK Data Processing software. The software fitted a freely jointed chain (FJC) model to each curve exhibiting an unbinding event.

$$
d(F)=L_{\mathrm{C}}\left[\operatorname{coth}\left(\frac{F l_{\mathrm{K}}}{k_{\mathrm{B}} T}\right)-\frac{k_{\mathrm{B}} T}{F l_{\mathrm{K}}}\right]
$$

where $L_{\mathrm{C}}$ is the contour length, $l_{\mathrm{K}}=700 \mathrm{pm}$ the Kuhn length, $k_{\mathrm{B}}$ the Boltzmann constant and $T$ the absolute temperature $[37,38]$. Furthermore, for each individual unbinding event, the software read out the unbinding force $F_{\mathrm{m}}$ and determined the loading rate $r$ by fitting the force versus time data just before the event.

\section{Results and Discussion}

\subsection{Fabrication of a SiNW Biosensor}

Figure 1 shows a scheme of the main steps to generate a SiNW field-effect transistor. First, gold microelectrodes were patterned by photolithography on a silicon-on-insulator sample (Figure 1a); o-SPL was applied to define a narrow and ultrathin silicon oxide mask (Figure 1b). A second photolithography step was applied to deposit a thin Au film and to close the gap between the microelectrodes and the o-SPL mask (Figure 1c). The next step involved the etching of the unmasked silicon region by reactive ion etching. Finally, the three electrodes of the back-gated device were connected to power sources (Figure 1d).

We developed a protocol for the selective deposition of the biomolecules on the SiNW surface. The process was based on controlling the adhesion force between the different surfaces and the proteins by using chemical functionalization and controlling the $\mathrm{pH}$ of the buffer [39]. The Au electrodes of the SiNW transistor (Figure 2a) were coated with a thin film of polydimethylsiloxane (Dow Corning's Sylgard Elastomer 184, purchased from Sigma Aldrich). This film was used to separate the Au electrodes from the solution (Figure 2b). Then, an OTS monolayer was deposited over the whole device (Figure 2c). The purpose of this layer was to avoid the unspecific binding of the proteins to the different surfaces of the device. The OTS monolayer was later removed from the SiNW surface by applying a large force with the AFM tip $(\sim 2 \mu \mathrm{N})$ (Figure $2 \mathrm{~d}$ ). Finally, the proteins (avidin) were deposited (Figure 2e). 


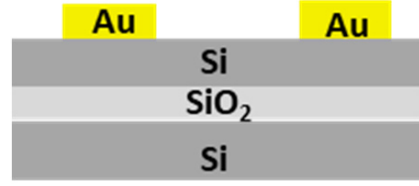

(a)

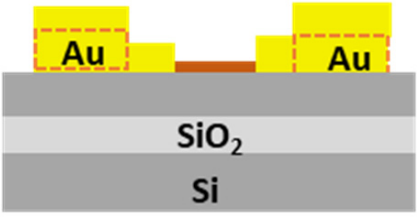

(c)

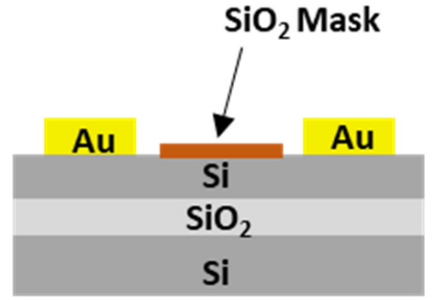

(b)

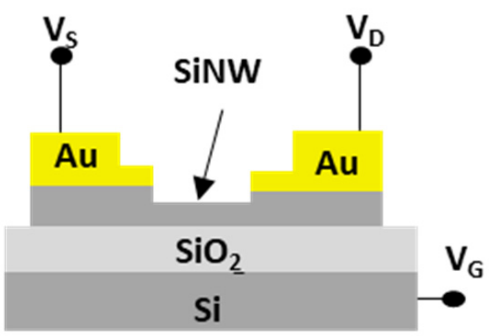

(d)

Figure 1. Main steps in the fabrication of a SiNW field-effect transistor by o-SPL. (a) Gold electrodes were defined by photolithography and electron beam evaporation. (b) Ultrathin and narrow oxide mask made by o-SPL. (c) Second photolithography step to connect the Au electrodes and the ultrathin oxide mask. (d) Reactive ion etching to remove the unprotected silicon regions. The etching also removed the ultrathin oxide mask. The final result was a back-gated Si nanowire FET.

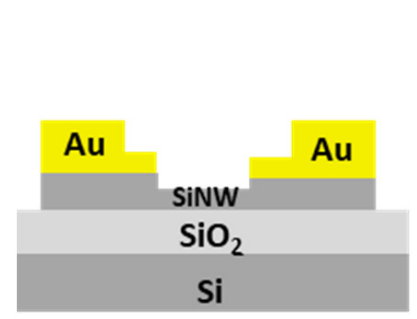

(a)

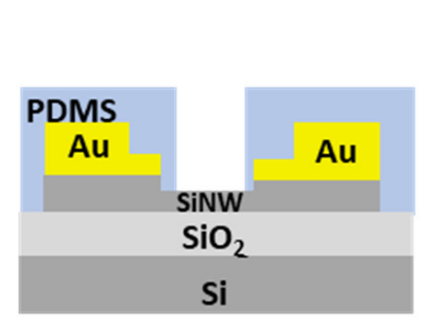

(b)

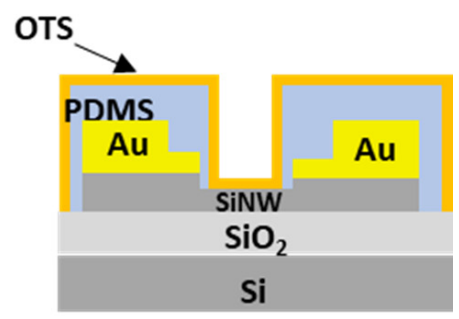

(c)

\section{- Biomolecules}

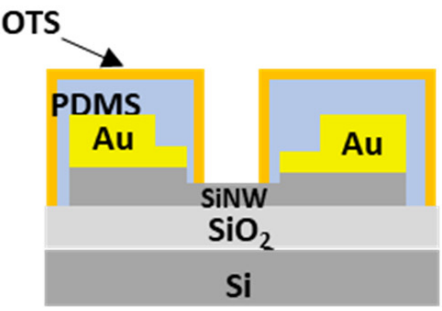

(d)

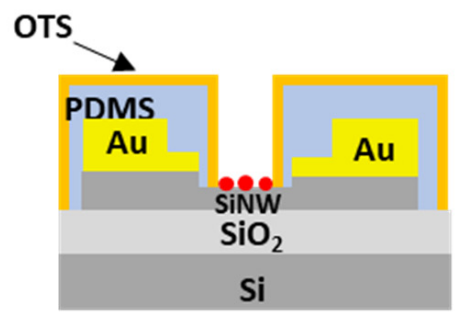

(e)

Figure 2. Steps to develop a SiNW biosensor. (a) SiNW FET. (b) PDMS deposition to protect the Au electrodes from the buffer solution. (c) Coating the device with an OTS layer. (d) Removal of the OTS from the surface of the SiNW by the AFM tip. (e) Adsorption and detection of biomolecules. 
Amplitude modulation AFM images of the surface of a SiNW were acquired before (Figure 3a) and after OTS functionalization and removal (Figure 3b). AFM phase images [33] obtained after the deposition of avidin revealed that the proteins were deposited on or near the SiNW surface (Figure 3c,d). From Figure 3d we were able to count the number of avidins over the nanowire. On average, one avidin molecule was found every $5 \mathrm{~nm}$ along the SiNW. An avidin molecule occupied approximately $30 \mathrm{~nm}^{2}$. In total, we estimated 10,000 molecules over the SiNW shown in Figure 3c.

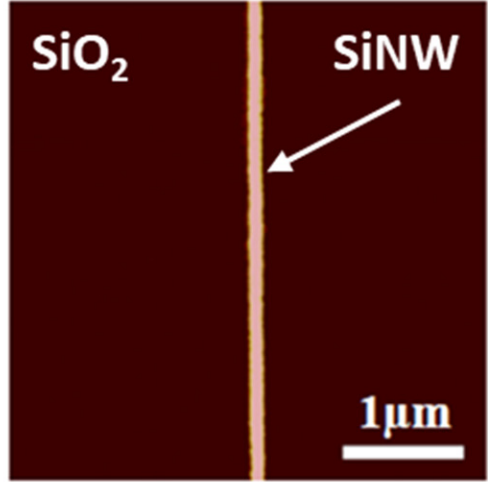

(a)

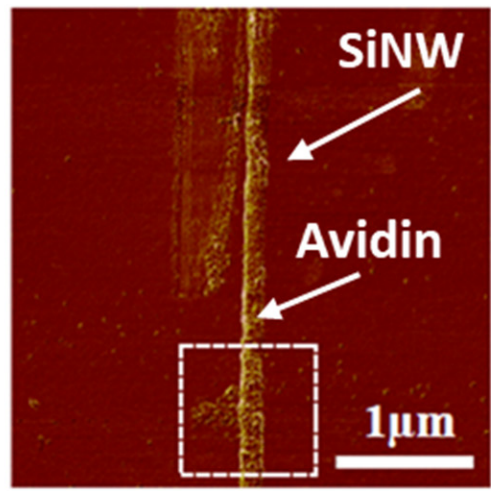

(c)

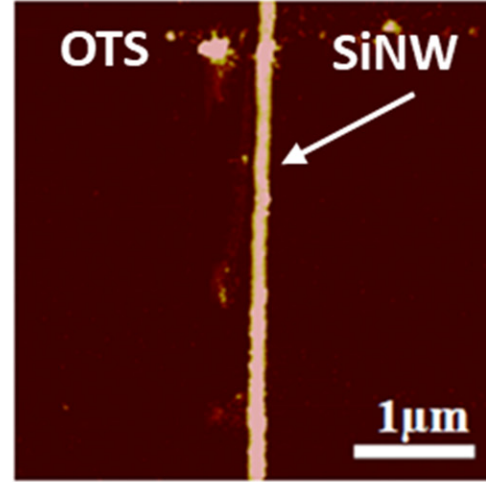

(b)

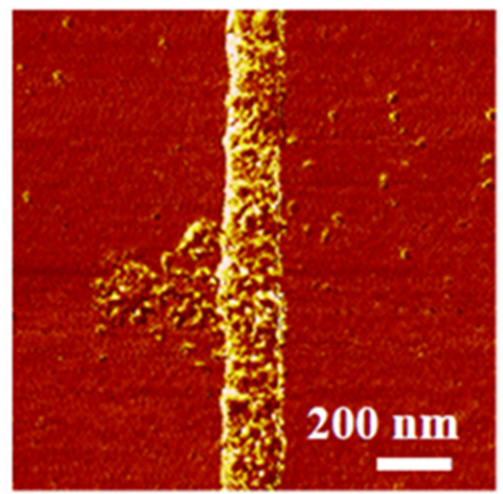

(d)

$95.1^{\circ}$

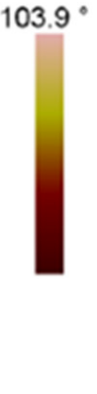

Figure 3. (a) AFM topographic image of a SiNW. (b) AFM topography of the same SiNW after OTS functionalization and removal. (c) AFM phase image after avidin molecules were deposited on the surface of the SiNW. (d) High resolution image of the area marked in (c). In this experiment, a $100 \mu \mathrm{L}$ drop containing avidin was deposited over the SiNW for $1 \mathrm{~min}$. Afterwards, the sample was rinsed with a $1 \mathrm{mM} \mathrm{NaCl}$ solution and, subsequently, with $0.02 \mathrm{M}$ PBS.

\subsection{Electrical Characterization of SiNW}

Figure $4 \mathrm{a}, \mathrm{b}$ showed the output curves of a SiNW FET in air and after its immersion in PBS (control measurements). The curves were very similar in both environments. This experiment showed the stability of the device in a liquid environment. Figure $4 \mathrm{c}$ showed the response of the same SiNW after a drop of a liquid containing avidin molecules was drop-casted. A significant increase of the current was observed (Figure 4c) with respect to the curves obtained in air or PBS. The increase of the current was consistent with the positive charge carried by avidin (the $\mathrm{pH}$ of the buffer was 7.4 while the isoelectric point of avidin was about 10). The corresponding transfer curves are shown in Figure $4 \mathrm{~d}-\mathrm{f}$. 


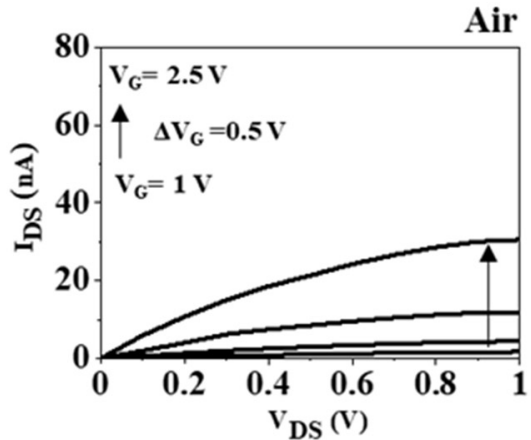

(a)

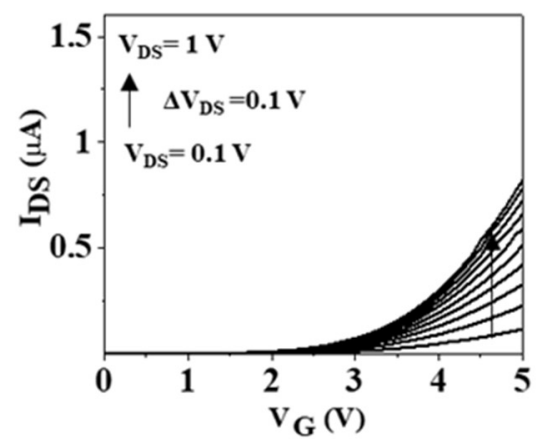

(d)

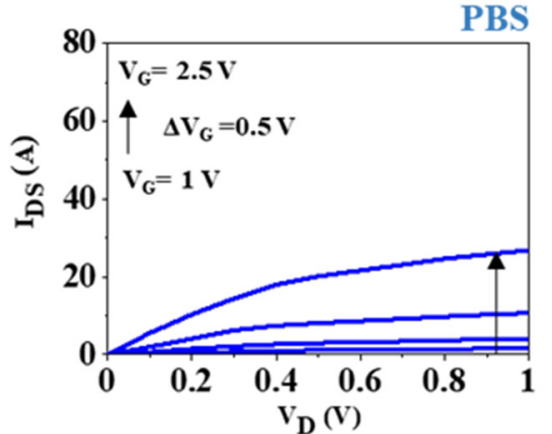

(b)

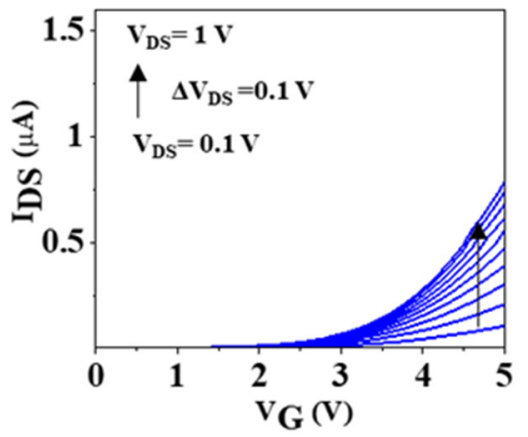

(e)

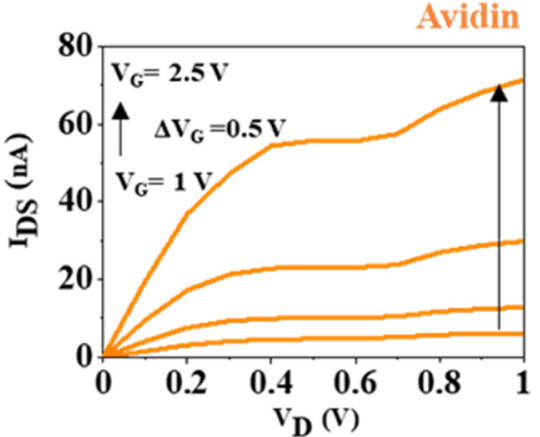

(c)

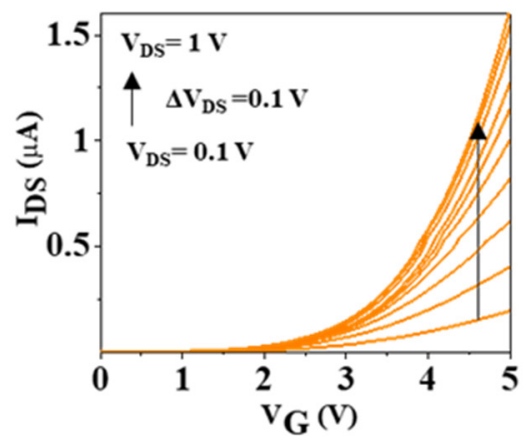

(f)

Figure 4. (a) Output curves of a SiNW transistor in air. (b) Output curves of the same transistor immersed in PBS. (c) Output curves of the same transistor after immersion in a liquid containing avidin molecules. (d) Transfer curves obtained from (a). (e) Transfer curves obtained from (b). (f) Transfer curves obtained from (c).

\subsection{Single-Molecule Force Spectroscopy on a SiNW}

Figure 5a shows the topography maps obtained from the SMFS force volume image. The image shows the SiNW in the center, surrounded by the OTS-covered substrate. Each pixel corresponds to an individual force-distance curve. While most of the retraction curves were featureless (Figure $5 b$ ), some curves exhibited a specific unbinding event. The positions of these curves are marked in blue. Light and dark blue corresponds to the OTS-covered substrate and the SiNW, respectively. Some example curves with specific unbinding events are shown in Figure 5c,d.

In total, 14 out of 800 force-distance curves showed specific events, from which 12 were located on the SiNW or right next to it. The SiNW had a width of approximately 6 pixels in the image, and consequently covered an area equivalent to 240 pixels. Thus, 240 forcedistance curves were obtained on the SiNW. Hence, on the SiNW, the chance to obtain a specific unbinding event was $5.0 \%(12 / 240)$, while on the surface covered by OTS it was $<0.4 \%(2 / 560)$. This result showed that the functionalization protocol significantly increased the chance for a target molecule (avidin) to be deposited on the SiNW's surface. The overall rate of recognition (5.0\%) was lower than some values reported elsewhere (around $27-29 \%)[30,40,41]$. The difference might be explained by the degrees of immobilization of the avidin. In previous works, the avidin was physisorbed to mica while here was bound to the SiNW. The stronger binding resulted in a lower flexibility of the avidin and hence reduced the probability that the biotin was bound to one of the avidin's four binding sites during the moment of contact. In any case, the key result here was that the binding probability found on the SiNW was one order of magnitude larger than the one found on the surfaces covered by OTS. A second experiment performed on a different SiNW confirmed the above finding, albeit the selectivity was slightly lower $(5.7 \%$ on the SiNW versus $1.0 \%$ on the OTS-covered substrate). 


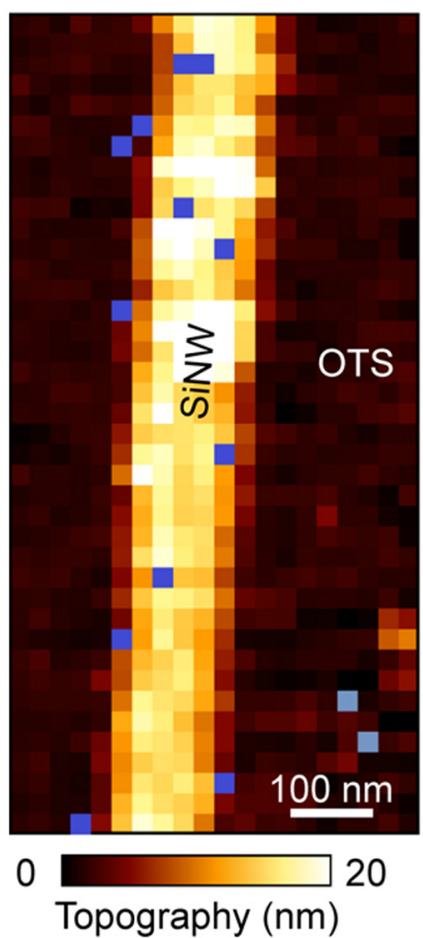

(a)
Specific events

- on SiNW

Total pixels:

Events:

next to SiNW

Total pixels:

Events:
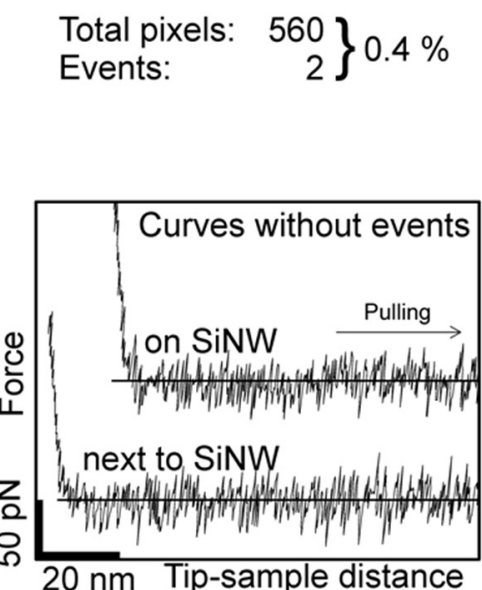

(b)

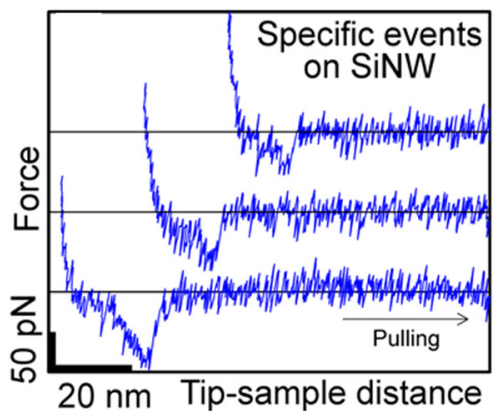

(c)

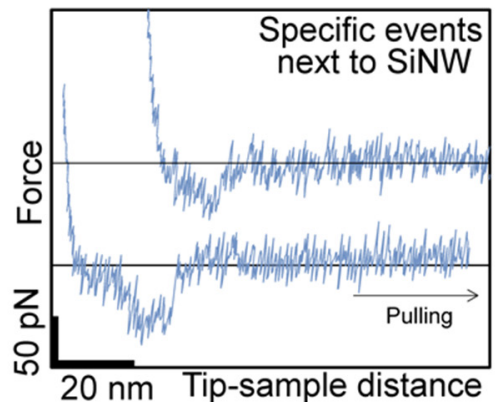

(d)

Figure 5. Detection of specific unbinding events on a silicon nanowire surrounded by an octadecyltrichlorosilane (OTS) layer. (a) AFM topography image obtained over the SiNW by force volume imaging. Each pixel corresponds to an entire force-distance curve. Pixels where specific avidin-biotin events were detected were marked in blue tones (blue: on the SiNW, light blue: next to the SiNW). (b) Examples of force-distance curves without unbinding events. (c) Examples of force-distance curves with specific unbinding events taken on the SiNW. (d) Force-distance curves with specific unbinding events taken on the OTS layer.

\section{Bell-Evans Model}

In order to verify the specificity of the observed unbinding events we applied the Bell-Evans model [42,43]. Figure 6a shows the overall distribution of force values. The average measured force, $F_{m}{ }^{*}=60.8 \mathrm{pN}$, agreed well with reported data obtained with cantilevers of similar stiffness [34,40].

A ligand-receptor system characterized by the presence of weak non-covalent bonds exhibits a dependence of the rupture forces (bond strengths) on the loading rate at which the force is applied [41]. The Bell-Evans theory provides the basic understanding of this dependency $[42,43]$. With increasing loading rate $r$, the mean value of the rupture force $F_{\text {rup }}$ increased according to:

$$
F_{\text {rup }}(r)=-\frac{k_{\mathrm{B}} T}{x_{\mathrm{u}}} \ln \left(\frac{r x_{\mathrm{u}}}{k_{\mathrm{off}} k_{\mathrm{B}} T}\right)
$$

where $k_{\text {off }}$ is the dissociation constant, $x_{\mathrm{u}}$ the effective distance between the bound and unbound states, $T$ the absolute temperature and $k_{\mathrm{B}}$ the Boltzmann constant. Figure $6 \mathrm{~b}$ showed the data from two experiments performed under similar conditions. The loading rate $r$ depended on both the pulling speed $v_{\mathrm{p}}$ and the effective linker stiffness, $k_{\text {eff }}$ according to $r=v_{\mathrm{p}} k_{\mathrm{eff}}$. The effective linker stiffness was related to the stiffness of the PEG-linker, $k_{\mathrm{PEG}}$, and the cantilever stiffness, $k_{0}$, by $k_{\mathrm{PEG}}=\frac{k_{0} k_{\text {eff }}}{k_{0}-k_{\text {eff }}}$. 


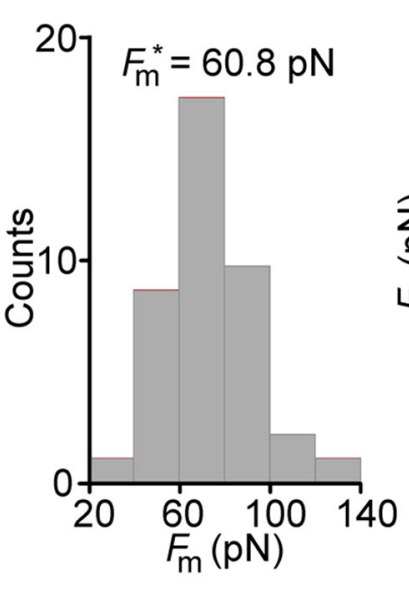

(a)

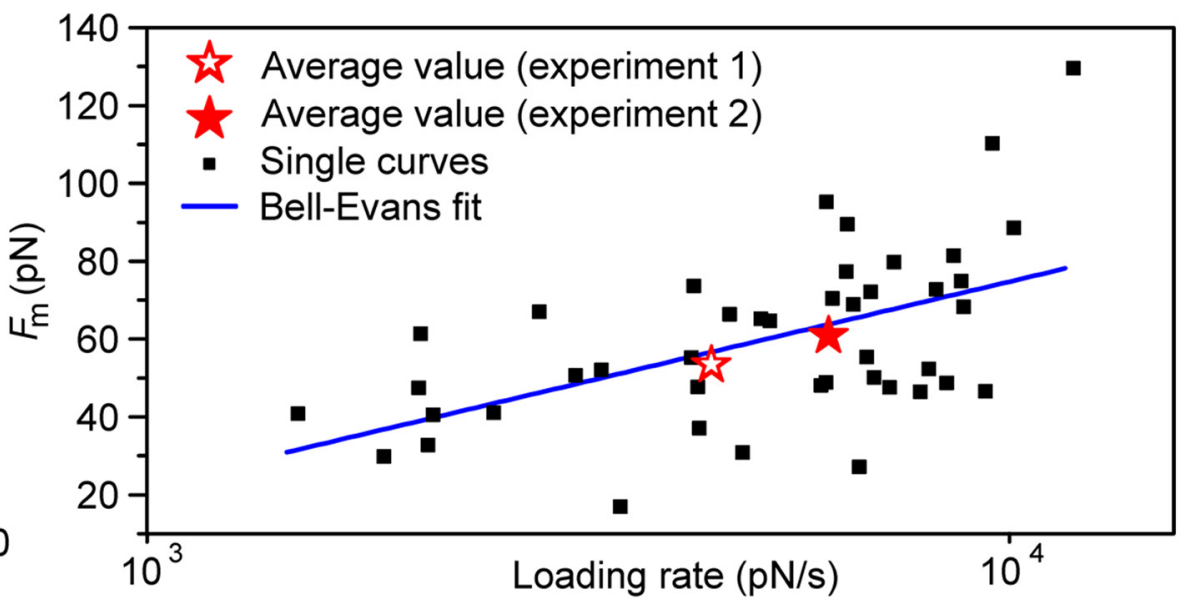

(b)

Figure 6. Quantitative analysis of the observed unbinding events. (a) Histogram of the measured forces $F_{m}$ with an average value of $F_{m}{ }^{*}=60.8 \mathrm{pN}$ (data from experiment 2). (b) Measured forces versus the loading rate. The data from all individual curves was shown (black squares) as well as the averaged values (red stars). The Bell-Evans fit including all black squares was shown in blue. The fit parameters were $\mathrm{x}_{\beta}=(0.21 \pm 0.06) \mathrm{nm}$ and $k_{\text {off }}=(21.4 \pm 14.1) \mathrm{s}-{ }^{1}$.

Although the experiments were performed using a constant pulling speed $v_{\mathrm{p}}$, the loading rate covered a range of values for two reasons. First, the cantilevers used for the two experiments had different stiffnesses $k_{0}$. Second, the stochastic nature of the unbinding process provided a range of rupture lengths, which, for a nonlinear spring, implied a distribution in the PEG stiffness [37].

The measured average force values were represented by the red stars. In order to increase the fit quality, it was useful to plot the individual data points instead of the average ones [44-46]. Fitting all data points using Equation (2) described the data well (both the average force values and the individual ones). The fit yielded $x_{\beta}=(0.21 \pm 0.06) \mathrm{nm}$ and $k_{\text {off }}=(21.4 \pm 14.1) \mathrm{s}^{-1}$ which was close to the values obtained previously for the inner activation barrier $[34,40]$.

Equation (2) was based on Hooke's law, which implies an accuracy limit for its application in AFM-based SMFS [47]. The validity of Equation (2) was verified by calculating the frequency ratio $x$ by [34].

$$
\chi=\frac{v_{\mathrm{p}}}{f_{1} x_{\mathrm{c}-\mathrm{c}}}
$$

For the measurement conditions used here, we obtained $\chi=\frac{1000 \mathrm{~nm} / \mathrm{s}}{3000 \mathrm{~s}^{-1} 0.15 \mathrm{~nm}} \approx 2.2$. Such a value of $\chi$ implied that the dynamic terms were very small and neglecting them led to an error of less than $1 \%$. Hence, the unbinding data was described accurately by Equation (2) (Bell-Evans model).

Altogether, it was concluded that the observed features were signatures of specific biotin-avidin unbinding events. The above results confirmed that the functionalization protocol of the SiNW provided a selective adsorption site for avidin molecules.

\section{Conclusions}

We aimed to correlate the indirect measurements of biomolecule concentrations obtained by SiNW biosensors with the direct molecular recognition data provided by singlemolecule force spectroscopy. To that end, we applied oxidation nanolithography, surface functionalization, electrical measurements and single-molecule force spectroscopy. A liquid containing avidin molecules increased the current through the SiNW device with respect to the control experiments. This was consistent with the positive charge carried by avidin. The 
SiNW field-effect transistor detected very low concentrations of avidin molecules (500 pM). In a subsequent experiment, we demonstrated the existence of specific molecular recognition events associated with the presence of avidin on the surface of the SiNW biosensor. Therefore, we confirmed that the nanoscale transistor measurements were correlated with the presence of avidin biomolecules on the surface of the nanowire.

Author Contributions: Conceptualization, R.G.; validation, F.M.E. and M.R.U.; writing-original draft preparation, F.M.E., M.R.U. and R.G.; supervision, R.G. All authors have read and agreed to the published version of the manuscript.

Funding: This research was funded by Ministerio de Ciencia e Innovación (PID2019-106801GB-I00), CSIC 202050E013, Comunidad de Madrid S2018/NMT-4443 (Tec4Bio-CM).

Conflicts of Interest: The authors declare no conflict of interest.

\section{References}

1. Sheehan, A.D.; Quinn, J.; Daly, S.; Dillon, P.; O'Kennedy, R. The Development of Novel Miniaturized Immuno-sensing Devices: A Review of a Small Technology with a Large Future. Anal. Lett. 2003, 36, 511-537. [CrossRef]

2. Lee, C.-S.; Kim, S.K.; Kim, M. Ion-Sensitive Field-Effect Transistor for Biological Sensing. Sensors 2009, 9, 7111-7131. [CrossRef]

3. Aspermair, P.; Ramach, U.; Reiner-Rozman, C.; Fossati, S.; Lechner, B.; Moya, S.E.; Azzaroni, O.; Dostalek, J.; Szunerits, S.; Knoll, W.; et al. Dual Monitoring of Surface Reactions in Real Time by Combined Surface-Plasmon Resonance and Field-Effect Transistor Interrogation. J. Am. Chem. Soc. 2020, 142, 11709-11716. [CrossRef]

4. Casalini, S.; Dumitru, A.C.; Leonardi, F.; Bortolotti, C.A.; Herruzo, E.T.; Campana, A.; De Oliveira, R.F.; Cramer, T.; Garcia, R.; Biscarini, F. Multiscale Sensing of Antibody-Antigen Interactions by Organic Transistors and Single-Molecule Force Spectroscopy. ACS Nano 2015, 9, 5051-5062. [CrossRef]

5. Cui, Y.; Wei, Q.; Park, H.; Lieber, C.M. Nanowire Nanosensors for Highly Sensitive and Selective Detection of Biological and Chemical Species. Science 2001, 293, 1289-1292. [CrossRef] [PubMed]

6. Chen, K.-I.; Li, B.-R.; Chen, Y.-T. Silicon nanowire field-effect transistor-based biosensors for biomedical diagnosis and cellular recording investigation. Nano Today 2011, 6, 131-154. [CrossRef]

7. Zhang, H.; Kikuchi, N.; Ohshima, N.; Kajisa, T.; Sakata, T.; Izumi, T.; Sone, H. Design and Fabrication of Silicon Nanowire-Based Biosensors with Integration of Critical Factors: Toward Ultrasensitive Specific Detection of Biomolecules. ACS Appl. Mater. Interfaces 2020, 12, 51808-51819. [CrossRef] [PubMed]

8. Zhang, G.-J.; Ning, Y. Silicon nanowire biosensor and its applications in disease diagnostics: A review. Anal. Chim. Acta 2012, 749, 1-15. [CrossRef] [PubMed]

9. Campbell, P.M.; Snow, E.S.; McMarr, P.J. Fabrication of nanometer-scale side-gated silicon field effect transistors with an atomic force microscope. Appl. Phys. Lett. 1995, 66, 1388-1390. [CrossRef]

10. Martinez, J.; Martinez, R.; Garcia, R. Silicon Nanowire Transistors with a Channel Width of 4 nm Fabricated by Atomic Force Microscope Nanolithography. Nano Lett. 2008, 8, 3636-3639. [CrossRef]

11. Martínez, R.V.; Martínez, J.; Garcia, R. Silicon nanowire circuits fabricated by AFM oxidation nanolithography. Nanotechnology 2010, 21, 245301. [CrossRef] [PubMed]

12. Chiesa, M.; Cardenas, P.P.; Otón, F.; Martinez, J.; Mas-Torrent, M.; Garcia, F.; Alonso, J.C.; Rovira, C.; Garcia, R. Detection of the Early Stage of Recombinational DNA Repair by Silicon Nanowire Transistors. Nano Lett. 2012, 12, 1275-1281. [CrossRef] [PubMed]

13. García, R.; Calleja, M.; Pérez-Murano, F. Local oxidation of silicon surfaces by dynamic force microscopy: Nanofabrication and water bridge formation. Appl. Phys. Lett. 1998, 72, 2295-2297. [CrossRef]

14. Ryu, Y.K.; Garcia, R. Advanced oxidation scanning probe lithography. Nanotechnology 2017, 28, 142003. [CrossRef]

15. Liu, S.; Maoz, R.; Sagiv, J. Planned Nanostructures of Colloidal Gold via Self-Assembly on Hierarchically Assembled Organic Bilayer Template Patterns with In-situ Generated Terminal Amino Functionality. Nano Lett. 2004, 4, 845-851. [CrossRef]

16. Garcia, R.; Tello, M.; And, J.F.M.; Biscarini, F. Size and Shape Controlled Growth of Molecular Nanostructures on Silicon Oxide Templates. Nano Lett. 2004, 4, 1115-1119. [CrossRef]

17. Ryu, Y.K.; Postigo, P.A.; Garcia, F.; Garcia, R. Fabrication of sub-12 nm thick silicon nanowires by processing scanning probe lithography masks. Appl. Phys. Lett. 2014, 104, 223112. [CrossRef]

18. Kurra, N.; Reifenberger, R.G.; Kulkarni, G.U. Nanocarbon-Scanning Probe Microscopy Synergy: Fundamental Aspects to Nanoscale Devices. ACS Appl. Mater. Interfaces 2014, 6, 6147-6163. [CrossRef]

19. Espinosa, F.M.; Ryu, Y.K.; Marinov, K.; Dumcenco, D.; Kis, A.; Garcia, R. Direct fabrication of thin layer MoS2 field-effect nanoscale transistors by oxidation scanning probe lithography. Appl. Phys. Lett. 2015, 106, 103503. [CrossRef]

20. Tomczyk, M.; Cheng, G.; Lee, H.; Lu, S.; Annadi, A.; Veazey, J.P.; Huang, M.; Irvin, P.; Ryu, S.; Eom, C.-B.; et al. Micrometer-Scale Ballistic Transport of Electron Pairs inLaAlO3/SrTiO3Nanowires. Phys. Rev. Lett. 2016, 117, 096801. [CrossRef] [PubMed] 
21. Liu, X.; Chen, K.; Wells, S.A.; Balla, I.; Zhu, J.; Wood, J.D.; Hersam, M.C. Scanning Probe Nanopatterning and Layer-by-Layer Thinning of Black Phosphorus. Adv. Mater. 2016, 29, 1604121. [CrossRef] [PubMed]

22. Dago, A.I.; Sangiao, S.; Fernández-Pacheco, R.; De Teresa, J.M.; Garcia, R. Chemical and structural analysis of sub-20 nm graphene patterns generated by scanning probe lithography. Carbon 2018, 129, 281-285. [CrossRef]

23. Zhao, P.; Wang, R.; Lien, D.; Zhao, Y.; Kim, H.; Cho, J.; Ahn, G.H.; Javey, A. Scanning Probe Lithography Patterning of Monolayer Semiconductors and Application in Quantifying Edge Recombination. Adv. Mater. 2019, 31, e1900136. [CrossRef]

24. Rani, E.; Wong, L.S. High-Resolution Scanning Probe Nanolithography of 2D Materials: Novel Nanostructures. Adv. Mater. Technol. 2019, 4, 1900181. [CrossRef]

25. Lipatov, A.; Li, T.; Vorobeva, N.S.; Sinitskii, A.; Gruverman, A. Nanodomain Engineering for Programmable Ferroelectric Devices. Nano Lett. 2019, 19, 3194-3198. [CrossRef]

26. Li, H.; Ying, Z.; Lyu, B.; Deng, A.; Wang, L.; Taniguchi, T.; Watanabe, K.; Shi, Z. Electrode-Free Anodic Oxidation Nanolithography of Low-Dimensional Materials. Nano Lett. 2018, 18, 8011-8015. [CrossRef]

27. Chen, L.; Li, J.; Tang, Y.; Pai, Y.-Y.; Chen, Y.; Pryds, N.; Irvin, P.; Levy, J. Extreme Reconfigurable Nanoelectronics at the CaZrO3/SrTiO3 Interface. Adv. Mater. 2018, 30, 1801794. [CrossRef]

28. Ryu, Y.; Dago, A.; He, Y.; Espinosa, F.; López-Elvira, E.; Munuera, C.; Garcia, R. Sub-10 nm patterning of few-layer MoS2 and MoSe2 nanolectronic devices by oxidation scanning probe lithography. Appl. Surf. Sci. 2020, 539, 148231. [CrossRef]

29. Borodin, B.R.; A Benimetskiy, F.; Alekseev, P.A. Study of local anodic oxidation regimes in MoSe2. Nanotechnology 2021, 32, 155304. [CrossRef] [PubMed]

30. Riener, C.K.; Stroh, C.M.; Ebner, A.; Klampfl, C.; Gall, A.A.; Romanin, C.; Lyubchenko, Y.L.; Hinterdorfer, P.; Gruber, H.J. Simple test system for single molecule recognition force microscopy. Anal. Chim. Acta 2003, 479, 59-75. [CrossRef]

31. Hutter, J.L.; Bechhoefer, J. Calibration of atomic-force microscope tips. Rev. Sci. Instrum. 1993, 64, 1868-1873. [CrossRef]

32. Garcia, R. Amplitude Modulation Atomic Force Microscopy; John Wiley \& Sons: Hoboken, NJ, USA, 2011.

33. Garcia, R. Nanomechanical mapping of soft materials with the atomic force microscope: Methods, theory and applications. Chem. Soc. Rev. 2020, 49, 5850-5884. [CrossRef] [PubMed]

34. Uhlig, M.R.; Amo, C.A.; Garcia, R. Dynamics of breaking intermolecular bonds in high-speed force spectroscopy. Nanoscale 2018, 10, 17112-17116. [CrossRef] [PubMed]

35. Dumitru, A.C.; Herruzo, E.T.; Rausell, E.; Ceña, V.; Garcia, R. Unbinding forces and energies between a siRNA molecule and a dendrimer measured by force spectroscopy. Nanoscale 2015, 7, 20267-20276. [CrossRef] [PubMed]

36. Duanis-Assaf, T.; Razvag, Y.; Reches, M. ForSDAT: An automated platform for analyzing force spectroscopy measurements. Anal. Methods 2019, 11, 4709-4718. [CrossRef]

37. Oesterhelt, F.; Rief, M.; E Gaub, H. Single molecule force spectroscopy by AFM indicates helical structure of poly(ethylene-glycol) in water. New J. Phys. 1999, 1, 6. [CrossRef]

38. Pfreundschuh, M.; Alsteens, D.; Wieneke, R.; Zhang, C.; Coughlin, S.R.; Tampé, R.; Kobilka, B.K.; Müller, D.J. Identifying and quantifying two ligand-binding sites while imaging native human membrane receptors by AFM. Nat. Commun. 2015, 6, 8857. [CrossRef]

39. Martínez, R.V.; Martínez, J.; Chiesa, M.; Garcia, R.; Coronado, E.; Pinilla-Cienfuegos, E.; Tatay, S. Large-scale Nanopatterning of Single Proteins used as Carriers of Magnetic Nanoparticles. Adv. Mater. 2010, 22, 588-591. [CrossRef]

40. De Paris, R.; Strunz, T.; Oroszlan, K.; Güntherodt, H.-J.; Hegner, M. Force Spectroscopy and Dynamics of the Biotin-Avidin Bond Studied by Scanning Force Microscopy. Single Molecules 2000, 1, 285-290. [CrossRef]

41. Bizzarri, A.R.; Cannistraro, S. Dynamic Force Spectroscopy and Biomolecular Recognition; Taylor and Francis: Abingdon, UK, 2012.

42. Bell, G.I. Models for the Specific Adhesion of Cells to Cells. Science 1978, 200, 618-627. [CrossRef]

43. Evans, E.; Ritchie, K. Dynamic strength of molecular adhesion bonds. Biophys. J. 1997, 72, 1541-1555. [CrossRef]

44. Alsteens, D.; Newton, R.; Schubert, R.; Martinez-Martin, D.; Delguste, M.; Roska, B.; Muller, D.J. Nanomechanical mapping of first binding steps of a virus to animal cells. Nat. Nanotechnol. 2016, 12, 177-183. [CrossRef]

45. Fuhrmann, A.; Ros, R. Single-molecule force spectroscopy: A method for quantitative analysis of ligand-receptor interactions. Nanomedicine 2010, 5, 657-666. [CrossRef] [PubMed]

46. Pfreundschuh, M.; Harder, D.; Ucurum, Z.; Fotiadis, D.; Müller, D.J. Detecting Ligand-Binding Events and Free Energy Landscape while Imaging Membrane Receptors at Subnanometer Resolution. Nano Lett. 2017, 17, 3261-3269. [CrossRef] [PubMed]

47. Amo, C.A.; García, R.G. Fundamental High-Speed Limits in Single-Molecule, Single-Cell, and Nanoscale Force Spectroscopies. ACS Nano 2016, 10, 7117-7124. [CrossRef] [PubMed] 